Proyecciones Journal of Mathematics

Vol. 36, No 1, pp. 187-194, March 2017.

Universidad Católica del Norte

Antofagasta - Chile

\title{
Jensen's and the quadratic functional equations with an endomorphism
}

\author{
KH. Sabour \\ IBN Tofail University, Morocco \\ and \\ S. Kabbaj \\ IBN Tofail University, Morocco \\ Received: October 2016. Accepted : October 2016
}

\begin{abstract}
We determine the solutions $f: S \rightarrow H$ of the generalized Jensen's functional equation

$$
f(x+y)+f(x+\varphi(y))=2 f(x), \quad x, y \in S,
$$

and the solutions $f: S \rightarrow H$ of the generalized quadratic functional equation

$$
f(x+y)+f(x+\varphi(y))=2 f(x)+2 f(y), \quad x, y \in S,
$$

where $S$ is a commutative semigroup, $H$ is an abelian group (2-torsion free in the first equation and uniquely 2-divisible in the second) and $\varphi$ is an endomorphism of $S$.
\end{abstract}

Subjclass[2010] : Primary 39B52.

Keywords : Functional equation, Jensen, quadratic, additive function, semigroup. 


\section{Set up, notation and terminology}

Throughout the paper we work in the following framework and with the following notation and terminology. We use it without explicit mentioning.

$S$ is a commutative semigroup [a set equipped with an associative composition rule $(x, y) \mapsto x+y], \varphi: S \rightarrow S$ is an endomorphism and $(H,+)$ denotes an abelian group with neutral element 0 . We say that $H$ is 2 -torsion free if $[\mathrm{h} \in H$ and $2 h=0] \Rightarrow h=0 . H$ is said to be uniquely 2-divisible if for any $h \in H$ the equation $2 x=h$ has exactly one solution $x \in H$.

A function $A: S \rightarrow H$ is said to be additive if $A(x+y)=A(x)+A(y)$ for all $x, y \in S$.

We recall that the Cauchy difference $C f$ of a function $f: S \rightarrow H$ is defined by

$$
C f(x, y):=f(x+y)-f(x)-f(y), \quad x, y \in S .
$$

\section{Introduction}

Let $\sigma \in \operatorname{Hom}(S, S)$ satisfy $\sigma^{2}=i d$. In [11], Sinopoulos determined the general solution $f: S \rightarrow H$, where $H$ is 2-torsion free, of Jensen's functional equation

$$
f(x+y)+f(x+\sigma(y))=2 f(x), \quad x, y \in S,
$$

and the general solution $f: S \rightarrow H$, where $H$ is uniquely 2-divisible, of the quadratic functional equation

$$
f(x+y)+f(x+\sigma(y))=2 f(x)+2 f(y), \quad x, y \in S .
$$

The purpose of the present paper is to solve the functional equations (2.1) and (2.2) on semigroups without using the condition $\sigma^{2}=i d$. More precisely, we solve the functional equations

$$
\begin{gathered}
f(x+y)+f(x+\varphi(y))=2 f(x), \quad x, y \in S, \\
f(x+y)+f(x+\varphi(y))=2 f(x)+2 f(y), \quad x, y \in S,
\end{gathered}
$$

where $\varphi$ is an endomorphism of $S$. By elementary methods we show that our formulas for the solutions of (2.3) (resp. (2.4)) on semigroups are the same as those of (2.1) (resp. (2.2)), so that our results constitute a natural extension of earlier results of, e.g., [11], from Jensen's (resp. the quadratic) functional equation with an involutive automorphism to that 
with an endomorphism. Note that the equation (2.3) has been solved in [3] under the assumption that $\varphi$ is surjective. Here we remove this restriction.

A similar functional equation that has been studied is

$$
f(x+y)+f(x+\varphi(y))=2 f(x) f(y), \quad x, y \in S,
$$

where $f: S \rightarrow C$ is the function to determine. Eq. (2.5) was solved in a more general framework (see [3]).

\section{The generalized Jensen's functional equation}

In this section, we solve the functional equation (2.3) by expressing its solutions in terms of additive functions.

Lemma 3.1. Let $f: S \rightarrow H$ be a solution of the functional equation (2.3). Then

$$
f\left(x+\varphi^{2}(y)\right)=f(x+y) \quad \text { for all } x, y \in S .
$$

Proof. Replacing $y$ by $\varphi(y)$ in (2.3), we get that

$$
f(x+\varphi(y))+f\left(x+\varphi^{2}(y)\right)=2 f(x) .
$$

Using this equation and (2.3), we obtain (3.1).

Lemma 3.2. Let $S$ be a semigroup (not necessarily abelian), $\phi: S \rightarrow S$ an endomorphism. If $f: S \rightarrow H$ and $\Phi: S \times S \rightarrow H$ satisfy

$$
f(x y)+f(\phi(y) x)=\Phi(x, y) \quad \text { for all } x, y \in S,
$$

then

$$
2 f(x y z)=\Phi(x, y z)-\Phi(\phi(z) x, y)+\Phi(x y, z) \quad \text { for all } x, y, z \in S
$$

Proof. Making the substitutions $(x, y z),(\phi(z) x, y)$, and $(x y, z)$ in $(3.2)$, we get respectively

$$
\begin{aligned}
f(x y z)+f(\phi(y z) x) & =\Phi(x, y z), \\
f(\phi(z) x y)+f(\phi(y z) x) & =\Phi(\phi(z) x, y) \\
f(x y z)+f(\phi(z) x y)) & =\Phi(x y, z) .
\end{aligned}
$$

Subtracting the middle identity from the sum of the other two we get (3.3). 
Theorem 3.3. Suppose that $H$ is 2-torsion free. The general solution $f: S \rightarrow H$ of the functional equation (2.3) is $f=A+c$, where $A: S \rightarrow H$ is an additive map such that $A \circ \varphi=-A$, and where $c \in H$ is a constant.

Proof. The method used here is closely related to and inspired by the one in [11, Proof of Theorem 2]. Assume that $f: S \rightarrow H$ is a solution of (2.3). Replacing $y$ by $y+\varphi(y)$ in (2.3) and using Lemma 3.1, we get

$$
f(x+y+\varphi(y))=f(x) .
$$

Using lemma 3.2 with $\Phi(x, y):=2 f(x)$, we find after division by 2 that

$$
\begin{aligned}
f(x+y+z) & =f(x)-f(\varphi(z)+x)+f(x+y) \\
& =f(x)-[f(x+z)+f(\varphi(z)+x)]+f(x+z)+f(x+y) \\
& =f(x+y)+f(x+z)-f(x) .
\end{aligned}
$$

Setting here $z=\varphi(x)$ and using (3.4), we get

$$
f(y)+f(x)=f(x+\varphi(x))+f(x+y) .
$$

Interchanging $x$ and $y$ in (3.5), we get that $f(x+\varphi(x))=f(y+\varphi(y))$ for all $x, y \in S$. So $f(x+\varphi(x))$ is a constant, say $c$. By using (3.5), we infer that the function $A(x):=f(x)-c$ is additive. Substituting $f$ into (2.3) we see that $A \circ \varphi=-A$.

The other direction of the proof is trivial to verify.

As an immediate consequence of Theorem 3.3, we have the following result.

Corollary 3.4 (2, Theorem 3.2). Suppose that $H$ is 2-torsion free and let $\sigma, \tau \in \operatorname{Hom}(S, S)$ such that $\sigma^{2}=\tau^{2}=i d$. The general solution $f: S \rightarrow$ $H$ of the functional equation $f(x+\sigma(y))+f(x+\tau(y))=2 f(x), \quad x, y \in S$, is $f=A+c$, where $A: S \rightarrow H$ is an additive map such that $A \circ \tau=-A \circ \sigma$, and where $c \in H$ is a constant.

Proof. The proof follows on putting $\varphi=\tau \circ \sigma$ in Theorem 3.3.

\section{The generalized quadratic functional equation}

In this section, we generalize Sinopoulos's result [11, Theorem 3] on semigroups by solving the functional equation (2.4). 
The following lemma lists pertinent basic properties of any solution $f: S \rightarrow H$ of $(2.4)$.

Lemma 4.1. Suppose that $H$ is 2-torsion free and let $f: S \rightarrow H$ be a solution of the functional equation (2.4).

(a) $f \circ \varphi=f$.

(b) For all $x, y, z \in S$, we have

$$
\begin{aligned}
f(x+y+z)= & f(x+y)+f(x+z) \\
& +f(y+z)-f(x)-f(y)-f(z) .
\end{aligned}
$$

(c) $C f: S \times S \rightarrow H$ is a symmetric, bi-additive map satisfying

$$
C f(x, \varphi(y))=-C f(x, y) \quad \text { for all } x, y \in S .
$$

(d) Let $A: S \rightarrow H$ be $A(x):=f(x+\varphi(x)), x \in S$. Then $A \circ \varphi=A$ and $A$ is additive.

(e) $2 f(x)=C f(x, x)+A(x)$ for all $x \in S$.

Proof. (a) Let us first observe that $f \circ \varphi$ is a solution of (2.4). We next replace $x$ by $\varphi(x)$ in (2.4) we find that

$$
f(\varphi(x)+y)+f(\varphi(x)+\varphi(y))=2 f(\varphi(x))+2 f(y) .
$$

Adding this equation to (2.4), we get

$$
\begin{aligned}
& {[f(x+y)+f(\varphi(x)+y)]+[f(x+\varphi(y))+f(\varphi(x)+\varphi(y))]} \\
& \quad=2 f(x)+2 f(\varphi(x))+4 f(y) .
\end{aligned}
$$

Using (2.4) and the fact that $H$ is 2-torsion free, we obtain

$$
[f(x)+f(y)]+[f(x)+f(\varphi(y))]=f(x)+f(\varphi(x))+2 f(y),
$$

i.e.

$$
f(x)-f \circ \varphi(x)=f(y)-f \circ \varphi(y) \quad \text { for all } x, y \in S .
$$

From this last equation we infer that $f-f \circ \varphi$ is a constant in $H$, say $c$. Using the fact that $f-f \circ \varphi$ is a solution of (2.4) and that $H$ is 2 -torsion free, we see that $c=0$. 
(b) Putting $\Phi(x, y):=2 f(x)+2 f(y)$ in lemma 3.2, we get after division by 2 that

$$
\begin{aligned}
f(x+y+z)= & f(x)+f(y+z)-f(\varphi(z)+x)-f(y)+f(x+y)+f(z) \\
= & f(x)+f(y+z)-[f(x+z)+f(\varphi(z)+x)]+f(x+z) \\
& -f(y)+f(x+y)+f(z) \\
= & f(x+y)+f(x+z)+f(y+z)-f(x)-f(y)-f(z),
\end{aligned}
$$

we get (4.1).

(c) That $C f$ is symmetric and bi-additive follows immediately from the very definition of $C f$ and (4.1). Let $x, y \in S$ be arbitrary. By help of (2.4) and $(a)$, we get that

$$
\begin{aligned}
C f(x, \varphi(y)) & =f(x+\varphi(y))-f(x)-f \circ \varphi(y) \\
& =2 f(x)+2 f(y)-f(x+y)-f(x)-f \circ \varphi(y) \\
& =f(x)+f(y)-f(x+y) \\
& =-C f(x, y) .
\end{aligned}
$$

(d) $\mathrm{A}$ is $\varphi$-even, because

$$
\begin{aligned}
A(\varphi(x)) & =f\left(\varphi(x)+\varphi^{2}(x)\right)=2 f \circ \varphi(x+\varphi(x)) \\
& =f(x+\varphi(x))=A(x) \quad \text { for all } x \in S .
\end{aligned}
$$

Next, let $x, y \in S$ be arbitrary. By help of (4.1) and (a), we find

$$
\begin{aligned}
A(x+y)= & f((x+\varphi(x))+y+\varphi(y)) \\
= & {[f(x+\varphi(x)+y)+f(x+\varphi(x)+\varphi(y))]+f(y+\varphi(y)) } \\
& -f(x+\varphi(x))-f(y)-f \circ \varphi(y) \\
= & 2 f(x+\varphi(x))+2 f(y)+f(y+\varphi(y))-f(x+\varphi(x)) \\
& -f(y)-f \circ \varphi(y) \\
= & f(x+\varphi(x))+f(y+\varphi(y)) \\
= & A(x)+A(y) .
\end{aligned}
$$

(e) Using (2.4), we obtain

$$
\begin{aligned}
C f(x, x)+A(x) & =f(x+x)+f(x+\varphi(x))-2 f(x) \\
& =4 f(x)-2 f(x)=2 f(x) \text { for all } x \in S .
\end{aligned}
$$

The second main theorem of the present paper reads as follows. 
Theorem 4.2. Suppose that $H$ is uniquely 2-divisible. The general solution $f: S \rightarrow H$ of the functional equation (2.4) is

$$
f(x)=Q(x, x)+A(x), \quad x \in S,
$$

where $Q: S \times S \rightarrow H$ is an arbitrary symmetric, bi-additive map such that $Q(x, \varphi(y))=-Q(x, y)$ for all $x, y \in S$, and where $A: S \rightarrow H$ is an arbitrary additive function such that $A \circ \varphi=A$.

Proof. That all solutions of (2.4) have this form is a consequence of Lemma 4.1 and the fact that $H$ is uniquely 2-divisible. Conversely, simple computations based on the properties of $Q$ and $A$, show that the indicated functions are solutions.

Acknowledgement. We wish to express our thanks to the referees for useful comments.

\section{References}

[1] B. Fadli, D. Zeglami and S. Kabbaj, On a Gajda's type quadratic equation on a locally compact abelian group, Indagationes Math. 26, pp. 660-668, (2015).

[2] B. Fadli, A. Chahbi, Iz. EL-Fassi and S. Kabbaj, On Jensen's and the quadratic functional equations with involutions, Proyecciones (Antofagasta) 35 (2), pp. 213-223, (2016).

[3] B. Fadli, S. Kabbaj, Kh. Sabour and D. Zeglami, Functional equations on semigroups with an endomorphism, Acta Math. Hungar. (2016), DOI 10.1007/s10474-016-0635-9.

[4] B. Fadli, D. Zeglami and S. Kabbaj, A variant of Jensen's functional equation on semigroups, Demonstratio Math., to appear.

[5] P. de Place Friis and H. Stetkær, On the quadratic functional equation on groups, Publ. Math. Debrecen 69, pp. 65-93, (2006).

[6] P. Kannappan, Functional equations and inequalities with applications, Springer, New York, (2009). 
[7] C.T. Ng, Jensen's functional equation on groups, Aequationes Math. 39, pp. 85-99, (1990).

[8] C.T. Ng, Jensen's functional equation on groups, II, Aequationes Math. 58, pp. 311-320, (1999).

[9] C.T. Ng, Jensen's functional equation on groups, III, Aequationes Math. 62, pp. 143-159, (2001).

[10] Th. M. Rassias, Inner Product Spaces and Applications, Pitman Research Notes in Mathematics Series, Addison Wesley Longman Ltd, 376, (1997).

[11] P. Sinopoulos, Functional equations on semigroups, Aequationes Math. 59, pp. 255-261, (2000).

[12] H. Stetkær, Functional equations on abelian groups with involution, Aequationes Math. 54, pp. 144-172, (1997).

[13] H. Stetkær, On Jensen's functional equation on groups, Aequationes Math. 66, pp. 100-118, (2003).

[14] H. Stetkær, Functional Equations on Groups, World Scientific Publishing Co, Singapore, (2013).

\section{KH. Sabour}

Department of Mathematics, Faculty of Sciences, IBN Tofail University, BP: 14000. Kenitra, Morocco

e-mail : khsabour2016@gmail.com

and

\section{S. Kabbaj}

Department of Mathematics, Faculty of Sciences,

IBN Tofail University, BP: 14000. Kenitra, Morocco e-mail : samkabbaj@yahoo.fr 